\title{
Elevated expression of miR-142-3p is related to the pro-inflammatory function of monocyte-derived dendritic cells in SLE
}

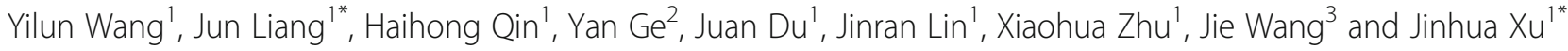

\begin{abstract}
Background: Recent studies have shown that alterations in the function of dendritic cells (DCs) are involved in the pathogenesis of systemic lupus erythematosus (SLE). However, the mechanism of the alteration remains unclear.

Methods: We cultured monocyte-derived DCs (moDCs) in vitro and examined the cytokines and chemokines in the supernatants of moDCs in negative controls (NC) and SLE patients in active phase. We then profiled microRNAs (miRNAs) of LPS-stimulated moDCs in SLE patients and used real-time PCR to verify the differentially expressed miRNAs. A lentiviral construct was used to overexpress the level of miR-142-3p in moDCs of NC. We examined the cytokines and chemokines in the supernatants of moDCs overexpressing miR-142-3p and used Transwell test, flow cytometric analysis and cell proliferation to observe the impact on $C D 4^{+} T$ cells in moDC-CD4 ${ }^{+} T$ cell co-culture.

Results: moDCs in patients with SLE secreted increased level of IL-6, CCL2 and CCL5, with attraction of more CD4 ${ }^{+}$ T cells compared with NC. We found 18 differentially expressed microRNAs in moDCs of SLE patients by microarray, and target gene prediction showed some target genes of differentially expressed miRNAs were involved in cytokine regulation. miR-142-3p was verified among the highly expressed miRNAs in the SLE group and overexpressing miR-142-3p in moDCs of the NC group caused an increase of SLE-related cytokines, such as CCL2, CCL5, CXCL8, IL-6 and TNF-a. Moreover, moDCs overexpressed with miR-142-3p resulted in attraction of an increased number of $C D 4^{+} T$ cells and in suppression of the proportion of Tregs in $D C-C D 4^{+} T$ cell co-culture whereas the proliferation of $C D 4^{+} T$ cells was not altered.
\end{abstract}

Conclusions: The results demonstrated a role for miR-142-3p in regulating the pro-inflammatory function of moDCs in the pathogenesis of SLE. These findings suggested that miR-142-3p could serve as a novel therapeutic target for the treatment of SLE.

Keywords: SLE, Monocyte-derived DCs, MicroRNA

\section{Background}

Systemic lupus erythematosus (SLE) is a complicated autoimmune disease impairing multiple organs. The disease predominantly affects women aged 15-40 years [1] with a female to male ratio of 9:1 [2]. Both genetic and environmental factors contribute to human SLE pathogenesis [3], but the etiology of SLE is not fully understood. Impaired clearance of dying cells may represent a central pathogenic process in human lupus [4]. The

\footnotetext{
* Correspondence: Liangjun1976@medmail.com.cn; xjhhuashan@126.com 'Department of Dermatology, Huashan Hospital, Fudan University, 12 Wulumuqi Zhong Road, Shanghai 200040, People's Republic of China Full list of author information is available at the end of the article
}

accumulated dying cells release autoantigens, which are presented by dendritic cells, further breaking down the immune tolerance of $\mathrm{T}$ and $\mathrm{B}$ cells and triggering SLE.

Dendritic cells (DCs) are so far the most potent antigenpresenting cells (APCs) with important functions in the immune system. Immunodysregulation in SLE involves the complex interplay of various immune cells and DCs are the master regulators for initiation, amplification, and perpetuation of the disease [5]. DCs could influence SLE in several ways including: presentation of self-antigens to autoreactive $\mathrm{T}$ cells; oversecretion of pro-inflammatory cytokines; and suppression of regulatory $\mathrm{T}$ cells and promotion of B cell autoantibody production, either directly 
or indirectly [6-8]. However, the mechanisms of the altered function of DCs in SLE are largely unknown.

MicroRNAs (miRNAs) are small noncoding RNAs, which regulate protein expression at the posttranscriptional level through reduction of mRNA stability or inhibition of translation via binding to the 3 ' un-translated regions (3'UTR) of target genes. They are involved in many kinds of diseases including tumors, infections, and autoimmune diseases. MicroRNAs can regulate DCs at different stages including differentiation, maturation, and apoptosis [9-12]. Monocyte-derived DCs (moDCs) from Blimp1 SLE-risk allele carriers exhibited increased expression of miRNA let-7c, which inhibited Blimp1 and also blocked lipopolysaccharide (LPS)-induced suppressor of cytokine signaling-1 (SOCS1) expression, contributing to the increased expression of pro-inflammatory cytokines [13]. The results suggested miRNAs in DCs might participate in the pathogenesis of SLE.

DC populations are relatively rare in blood. We therefore utilized the well-accepted model of human DC differentiation from peripheral blood monocytes under inflammatory conditions, which is a useful tool to study DC functions ex vivo [14-16]. Pathogenic inflammation can trigger SLE disease exacerbations [17, 18], in which Tolllike receptor 4 (TLR4) and TLR4 responsiveness are important [19]. We therefore choose LPS-activated moDCs as a subtype of DCs generated upon inflammation.

The aim of our study was to find any miRNAs participating in regulating the function of moDCs in SLE. In the study, we found moDCs in the SLE group produced increased levels of pro-inflammatory cytokines. Since all culture conditions were the same, the discrepancy between the SLE group and healthy control group was due to the intrinsic factors. MiRNA microarray analysis was conducted and the target genes of miRNAs significantly differentially expressed in patients with SLE were involved in cytokine regulation through target gene prediction. We further proved the expression level of miR-142$3 p$ might influence the function of moDCs. Thus, we proposed a regulatory mechanism of miR-142-3p in moDCs of SLE.

\section{Methods}

\section{Subjects}

Fifteen female SLE patients whose SLE Disease Activity Index (SLEDAI) score was moderate to severe were recruited from the inpatient service in Huashan Hospital, Fudan University. Relevant clinical and laboratory information regarding the patients is shown in Table 1. The diagnostic criteria were in accordance with the 1997 American College of Rheumatology (ACR) revised criteria for the classification of SLE. Fifteen female healthy controls were also recruited. The study was approved by the Independent Ethics Committee of Huashan Hospital
Table 1 Clinical and laboratory characteristics of the patients with SLE in the study

\begin{tabular}{ll}
\hline Characteristics & $\operatorname{SLE}(\mathrm{n}=15)$ \\
\hline Sex [male/female (n)] & $0 / 15(15)$ \\
Age (years) [median (range)] & $33(17-57)$ \\
SLEDAl score [median (range)] & $15(10-30)$ \\
Anti-dsDNA (IU/ml) [median (range)] & $391.9(25.9-800)$ \\
C3 (g/l) [median (range)] & $0.6(0.22-1.02)$ \\
C4 (g/l) [median (range)] & $0.07(0.06-0.15)$ \\
Red cell count $\left({ }^{*} 10 \wedge 12 / l\right)[$ median (range)] & $3.91(3.16-4.44)$ \\
Lymphocyte count $\left({ }^{*} 10 \wedge 9 / /\right)$ [median (range)] & $4.74(1.56-19.78)$ \\
Platelet count $\left({ }^{*} 10 \wedge 9 / /\right)[$ median (range)] & $181(25-382)$ \\
Organ involvement $[\mathrm{Y} / \mathrm{N}(\mathrm{n})]$ & $6 / 9(15)$ \\
Steroids $[\mathrm{Y} / \mathrm{N}(\mathrm{n})]$ & $9 / 6(15)$ \\
Immunosuppressant $[\mathrm{Y} / \mathrm{N}(\mathrm{n})]$ & $0 / 15(15)$ \\
\hline
\end{tabular}

SLE systemic lupus erythematosus, SLEDAI SLE Disease Activity Index, dsDNA double-stranded DNA

and written informed consent was obtained from all subjects. All the experiments were carried out in accordance with the relevant guidelines and regulations of Huashan Hospital.

\section{Cell culture}

EDTA blood $(25 \mathrm{ml})$ was collected from each patient and control subject. Nearly $1-2^{*} 10^{\wedge} 7$ peripheral blood mononuclear cells (PBMCs) were isolated from each patient's blood and $\mathrm{CD} 14^{+}$monocytes were sorted by positive selection (purity $>90 \%$ ) using magnetic beads (Miltenyi Biotec, Bergisch Gladbach, Germany). CD14 $4^{+}$ monocytes were then cultured for 5-7 days in RPMI 1640 containing $10 \%$ fetal bovine serum (FBS), penicillin/streptomycin solution and L-glutamine (Life Technologies, Carlsbad, CA, USA) supplemented with 1000 $\mathrm{U} / \mathrm{ml}$ granulocyte-macrophage colony-stimulating factor (GM-CSF) and $1000 \mathrm{U} / \mathrm{ml}$ interleukin-4 (IL-4) every 2 days (PeproTech, Rocky Hill, NJ, USA). For transfection experiments, immature moDCs were harvested at day 5. For moDC maturation, $1 \mu \mathrm{g} / \mathrm{ml}$ LPS (Escherichia coli type 055:B6; Sigma-Aldrich, St. Louis, MO, USA) was added into the medium at day 6 . The following antibodies were used to identify the phenotype of moDCs: anti-CD11c, anti-HLA-DR, anti-CD40, anti-CD86, antiCD83 and low expression of anti-CD14 (eBioscience, San Diego, CA, USA).

\section{Lentiviral transfections}

A fragment encoding miR-142-3p was amplified by PCR from human genomic DNA using the primers $5^{\prime}$ GCCACAAGGAGGGCTGGGGGGC-3' and $5^{\prime}$-GAG CGCCGAGGAAGATGGTGGC-3'. Those fragments confirmed by sequencing were cloned into the pHBLV 
vector respectively (Hanbio, Shanghai, China). moDCs transfected with the empty lentiviral vector (designated as VEC) or RPMI 1640 medium (designated as NC) were used as controls. After 3 days, the transfected moDCs were collected.

\section{Microarray analysis and real-time PCR}

Total RNA was extracted from moDCs using the miRNeasy Mini Kit (Qiagen, Hilden, Germany). miRNA expression profiling was determined by miRNA microarray analysis using the Agilent Human miRNA Array V19.0 ID:046064 (Agilent Technologies, Santa Clara, CA, USA) that included 2006 mature human miRNAs. Differentially expressed miRNAs were identified using the paired $t$ test with the cutoff criteria of $P<0.05$.

Reverse transcription was performed to obtain the cDNA for miRNA using the All-in-One miRNA qRTPCR Detection Kit (Genecopoiea, Rockville, MD, USA). Quantitative real-time PCR was carried out with the Rotor-Gene Q (Qiagen) using the All-in-One miRNA qRT-PCR Detection Kit (Genecopoiea). The housekeeping gene U6 was used as the internal control. The primers for microRNAs and U6 were purchased from Genecopoiea directly.

\section{Target gene prediction}

The target genes of differentially expressed miRNAs were predicted by at least two databases of the following five usual prediction databases: TargetScan (http:// www.targetscan.org), miRanda (http://www.microrna.org/ microrna/home.do), PicTar (http://pictar.mdc-berlin.de/), MirTarget2 from miRDB (http://mirdb.org/miRDB/ down load.html), and PITA (http://genie.weizmann.ac.il). Moreover, the Gene Ontology (GO) functional and pathway enrichment analysis were conducted for the target genes using the Database for Annotation, Visualization and Integrated Discovery (DAVID) online tools with the cutoff criterion of a false discovery rate $(\mathrm{FDR})<0.05$.

\section{moDCs-CD4 ${ }^{+} \mathrm{T}$ cells co-culture}

moDCs in each group were collected and overloaded with OVA peptide (Sigma-Aldrich) for $2 \mathrm{~h}$, used as stimulator cells. They were suspended in RPMI 1640 medium to a final concentration of $5 \times 10^{5} / \mathrm{ml}$. Allogeneic $\mathrm{CD}^{+}{ }^{+} \mathrm{T}$ cells were obtained from positive selection of PBMCs as responding cells. The density of responding $\mathrm{CD}^{+} \mathrm{T}$ cells was adjusted to $5 \times 10^{6} / \mathrm{ml}$. Stimulator cells and responding cells were added to each well on the 96-well plates. Each sample was tested in triplicate. The stimulator and responding cells were cultured together in an incubator $\left(37^{\circ} \mathrm{C}, 5 \% \mathrm{CO}_{2}\right)$ for 3 days.

\section{$\mathrm{CD}^{+}{ }^{+} \mathrm{CD} 25^{+} \mathrm{Foxp}^{+}{ }^{+}$Tregs analysis}

After co-culture for 3 days, cell suspensions were incubated with FITC-conjugated anti-human CD4 and PEconjugated anti-human CD25 (Biolegend, San Diego, CA, USA) for $30 \mathrm{~min}$ at $4{ }^{\circ} \mathrm{C}$ and washed twice with $2 \mathrm{ml}$ of phosphate-buffered saline (PBS) $\mathrm{pH} 7.4$ containing $1 \%$ bovine serum albumin (BSA). Intracellular staining for Foxp3 was then performed with APCconjugated anti-human Foxp3 (eBioscience, San Diego, CA, USA) for $60 \mathrm{~min}$ and then washed with PBS/BSA. The supernatants were discarded and cells were resuspended in $0.2 \mathrm{ml}$ PBS/BSA. Data were acquired with a FACSCanto system (Becton Dickinson, Franklin Lakes, NJ, USA) and analyzed using Flowjo software (Tree Star, Inc., Ashland, OR, USA). The expression levels of CD4, CD25 and Foxp3 were evaluated by calculating the percentage of cells expressing each protein.

\section{$\mathrm{CD}^{+} \mathrm{T}$ cells proliferation}

$\mathrm{CD} 4^{+} \mathrm{T}$ cells were labeled with $5 \mu \mathrm{M}$ carboxyfluorescein diacetate succinimidyl ester (CFSE, Molecular Probes, Eugene, OR, USA) first and then co-cultured with moDCs. After 3 days, CFSE dilution was analyzed using flow cytometric analysis. The proliferation experiment was evaluated using a division index in Flowjo software (Tree Star, Inc.).

\section{Chemotaxis assay}

$\mathrm{CD}^{+} \mathrm{T}$ cells were placed on the upper chamber of a Transwell plate, $6.5 \mathrm{~mm}$ in diameter, with $5-\mu \mathrm{m}$ polycarbonate filters (Corning, Corning, NY, USA). The lower chamber contained either diluted moDC supernatant (1:1 with medium) or control medium. After culture of $3 \mathrm{~h}$ at $37{ }^{\circ} \mathrm{C}$, the cells that had migrated to the lower chamber were harvested and counted under a light microscope.

\section{Chemokine and cytokine assays}

Chemokines [C-X-C motif ligand (CXCL)8, C-C motif ligand (CCL) 2 and CCL5] and cytokines [IL-6, tumor necrosis factor alpha (TNF- $\alpha$ ), IL-10 and IL-17] in supernatants of moDCs or supernatants of $\mathrm{CD} 4^{+} \mathrm{T}$ cells-moDCs co-culture were simultaneously quantified using the Cytometric Bead Array (CBA) reagent kits (BD Biosciences Pharmingen, San Diego, CA, USA).

\section{Statistics}

Continuous variables were expressed as mean (SD) and categorical variables as frequencies (\%). The Student $t$ test or one-way analysis of variance was used to compare continuous variables. All $P$ values were estimated in a two-tailed fashion. Differences were considered to be statistically significant at $P<0.05$. Data were analyzed using SPSS 13.0 (SPSS Inc., Chicago, IL, USA). 


\section{Results}

Pro-inflammatory function of moDCs in SLE

Since cytokine production was one of the major functions of DCs with great biological importance, we first investigated whether the secreted concentration of some cytokines and chemokines in the supernatants of moDCs was different between SLE patients and healthy controls. The result showed mature moDCs from patients with SLE produced significantly higher levels of IL-6, CCL2 and CCL5 compared with mature moDCs from healthy controls (Fig. $1 \mathrm{a}-\mathrm{c}$ ). We also measured levels of TNF- $\alpha$ and CXCL8 in cell culture supernatants between the two groups without finding significant differences (Fig. 1d and e). In addition, infiltration of $\mathrm{T}$ lymphocytes and other leukocytes into the sites of inflammation is important in SLE. We then found that supernatants of moDCs in the SLE group attracted significantly more allogeneic $\mathrm{CD}^{+}{ }^{+} \mathrm{T}$ cells than the control group and culture medium group though a Transwell assay (Fig. 1f).

miRNA profiling in LPS-activated moDCs of patients with SLE miRNAs were able to regulate the function of DCs and we therefore investigated whether these noncoding RNAs would impact the function of moDCs in patients with SLE.
We first performed miRNA microarrays to identify the expression levels of matured miRNAs purified from 24-h LPS-activated moDCs from five SLE patients who were in the active phase of the disease and five negative controls. Microarrays identified 18 miRNAs that were differentially regulated in SLE (Fig. 2). Table 2 showed the fold change and $P$ value of the differentially regulated miRNAs. In all, the expression of eight miRNAs (miR-142-3p, miR-551b3p, miR-3127-5p, miR-671-5p, miR-630, miR-5703, miR6125, and miR-574-5p) was significantly increased in the patients with SLE, whereas the expression of ten miRNAs (miR-338-5p, let-7i-3p, miR-181b-5p, miR-1260b, miR125a-5p, miR-1260a, miR-15b-5p, miR-25-3p, miR-26a-5p, and miR-564) was significantly decreased.

Next, we performed functional and pathway enrichment analysis for target genes of all the differentially expressed miRNAs. The top ten functional and pathway enrichment analysis results are indicated in Additional files 1 and 2 respectively.

\section{Validation of differentially expressed miRNAs in the SLE group}

Seven differentially expressed miRNAs (miR-142-3p, miR630, miR-671-5p, miR-15b-5p, miR-181b-5p, miR-125a-

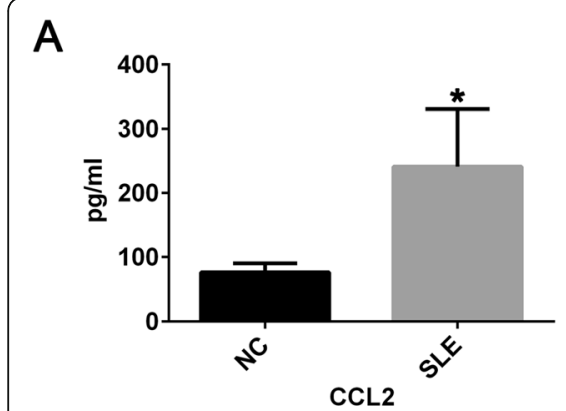

D

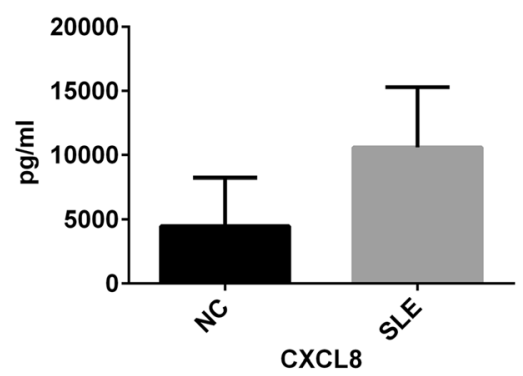

B

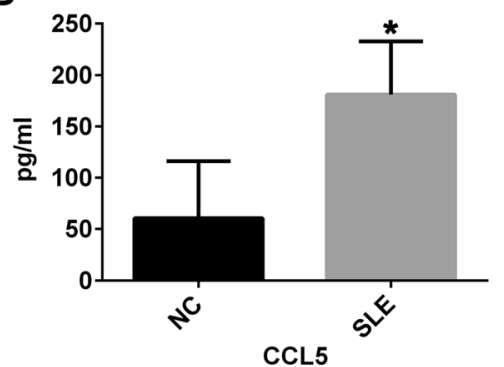

E

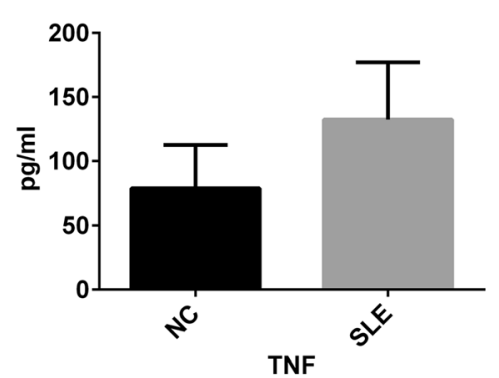

C

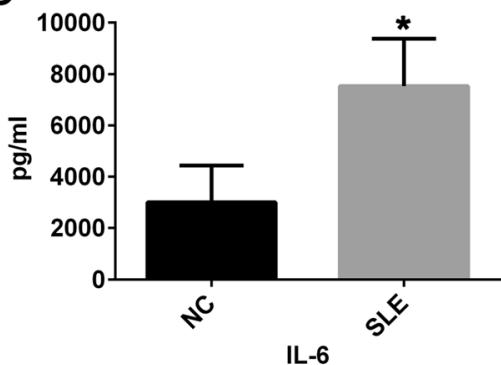

F

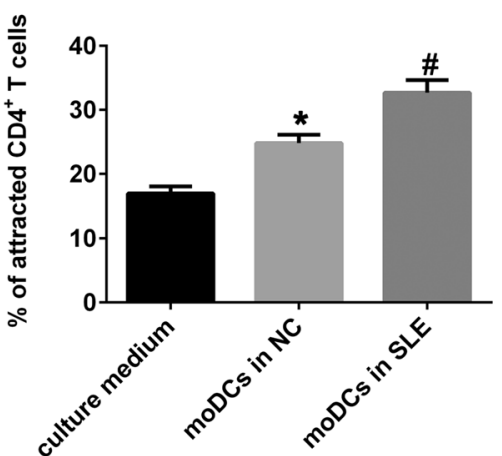

Fig. 1 Pro-inflammatory function of moDCs in SLE. The supernatants derived from culture medium of moDCs from negative controls $(n=5)$ and patients with SLE $(n=5)$ in the presence of LPS $(1 \mu \mathrm{g} / \mathrm{ml})$ for 24 hours was assessed using cytometric bead array including CCL2 $\left(\mathbf{a},{ }^{*} P<0.001\right)$, CCL5 (b, $\left.{ }^{*} P=0.002\right), I L-6$ (c, $\left.{ }^{*} P=0.002\right), C X C L 8$ (d, no significant difference) and TNF-a (e, no significant difference). Percentage of CD4 ${ }^{+} \mathrm{T}$ cells attracted by supernatants of culture medium, supernatants of moDCs in NC, and supernatants of moDCs in SLE group ( $\mathbf{f}_{,}{ }^{*} P<0.05$ versus supernatants of culture medium and supernatants of moDCs in $N C$, ${ }^{*} P<0.05$ versus supernatants of culture medium). Data were shown as mean $\pm S D$. CCL C-C motif ligand, CXCL C-X-C motif ligand, IL interleukin, moDCs monocyte-derived DCs, NC negative controls, SLE systemic lupus erythematosus, TNF-a tumor necrosis factor alpha 


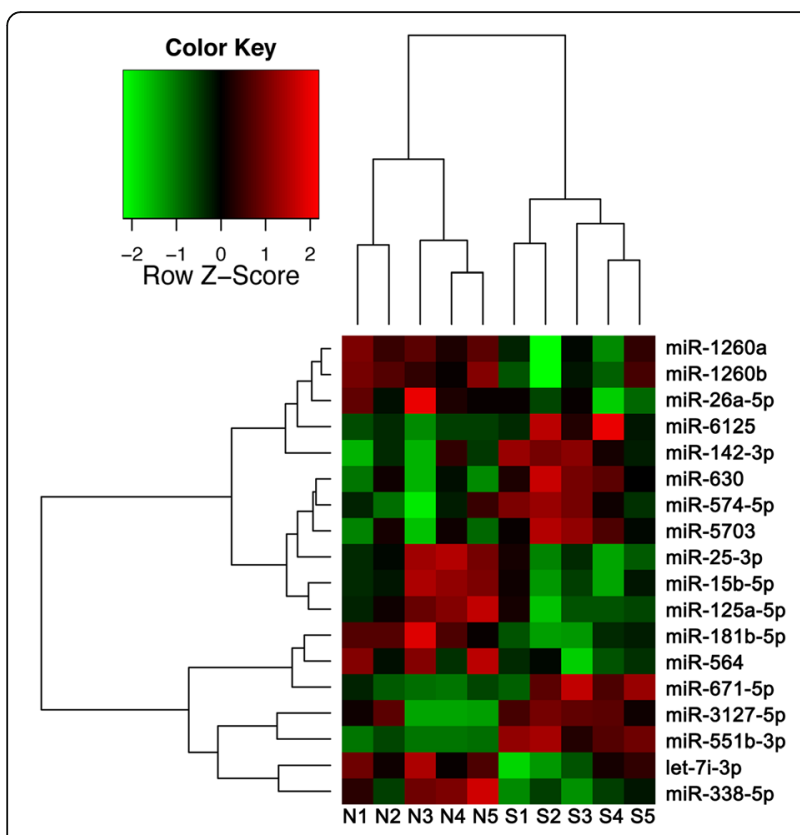

Fig. 2 miRNA profiling in LPS-activated moDCs of patients with SLE. The expression levels of mature miRNAs purified from 24-h LPSactivated moDCs were analyzed using miRNA microarrays and hierarchical clustering of statistically significant differential miRNAs with analysis of variance $(P<0.05)$. N $=10$. N1-N5 represented negative controls. S1-S5 represented the patients with SLE

Table 2 Fold change and $P$ values of differentially expressed miRNAs with $P<0.05$

\begin{tabular}{lll}
\hline Expression miRNA in patients of SLE & Fold change & $P$ value \\
\hline Increased & & \\
hsa-miR-551b-3p & 55.1 & $<0.001$ \\
hsa-miR-3127-5p & 3.3 & 0.044 \\
hsa-miR-671-5p & 2.7 & 0.024 \\
hsa-miR-630 & 2.2 & 0.010 \\
hsa-miR-5703 & 2.1 & 0.023 \\
hsa-miR-6125 & 1.5 & 0.047 \\
hsa-miR-142-3p & 1.5 & 0.021 \\
hsa-miR-574-5p & 1.4 & 0.048 \\
Decreased & & \\
hsa-miR-338-5p & 0.1 & 0.014 \\
hsa-let-7i-3p & 0.2 & 0.036 \\
hsa-miR-181b-5p & 0.4 & 0.003 \\
hsa-miR-1260b & 0.6 & 0.042 \\
hsa-miR-125a-5p & 0.6 & 0.017 \\
hsa-miR-1260a & 0.6 & 0.042 \\
hsa-miR-15b-5p & 0.7 & 0.028 \\
hsa-miR-25-3p & 0.8 & 0.020 \\
hsa-miR-26a-5p & 0.8 & 0.042 \\
hsa-miR-564 & 0.9 & 0.030 \\
\hline miRNA microRNA, SLE systemic lupus erythematosus &
\end{tabular}

miRNA microRNA, SLE systemic lupus erythematosus 5p, and miR-5703) whose target genes were significantly enriched in important functions and pathways were selected for further validation. We used quantitative realtime PCR (qRT-PCR) to validate the microarray findings among 15 patients and 15 healthy controls including those five patients and five healthy controls used in the microarray analysis (Fig. 3). The result of qRT-PCR revealed that six miRNAs (miR-142-3p, miR-630, miR-15b-5p, miR181b-5p, miR-125a-5p, and miR-5703) showed the same change patterns as shown in the microarray analysis. However, the expression level of miR-671-5p was significantly decreased to 0.81 -fold of NC $(P=0.021)$, which was opposite to the microarray analysis (Fig. 3h). This discrepancy might be due to technical limitations of the microarray, such as cross-hybridization, signal saturation, and limited dynamic range.

Among all the differentially expressed miRNAs, miR142-3p attracted our attention. miR-142-3p was demonstrated to be essential for the specification of the hemangioblastic precursors of the blood stem cell lineage and might facilitate hematopoietic stem cells formation [20]. It was reported that miR-142/- mice developed abnormal hematopoietic lineages [21], and displayed an impairment of $\mathrm{CD}^{+}{ }^{+} \mathrm{DC}$ homeostasis both in vitro and in vivo, leading to a severe and specific defect in the priming of $\mathrm{CD}^{+} \mathrm{T}$ cells [22]. On the other hand, the enhanced expression of miR-142-3p reduced the immunosuppressive activity of bone marrow myeloid-derived suppressor cells, restored $\mathrm{CD} 8^{+} \mathrm{T}$ cell proliferation and was able to change the ratio of macrophage: $\mathrm{DC}$ to favor the DC expansion [23]. In our study, we found miR-142$3 p$ in moDCs was upregulated 2.33-fold in SLE patients $(P<0.001)$ (Fig. 3a), and moDCs of SLE patients secreted increased pro-inflammatory cytokines together with attracting more $\mathrm{CD} 4^{+} \mathrm{T}$ cells. Our functional enrichment analysis also indicated that target genes of miR-142-3p were involved in cytokine and chemokine regulation. As a result, we hypothesized that overexpression of miR$142-3 p$ in moDCs might impact the function of moDCs and then lead to overactive immune reaction in the pathogenesis of SLE. We thus selected miR-142-3p for further study.

\section{Overexpression of miR-142-3p promoted pro-inflammatory function of moDCs}

We investigated the potential role of miR-142-3p in moDCs through regulating the basal expression level of miR-142-3p. Transfection efficiency was determined by observing the fluorescent cells (Fig. 4a) and expression level of miR-142-3p in moDCs by qRT-PCR. The expression of miR-142-3p increased 4.79-fold in the miR-142-3p lentivirus (LV) group compared with the empty lentiviral vector (VEC) group while no significance was found between the VEC and NC group (Fig. 4b). 


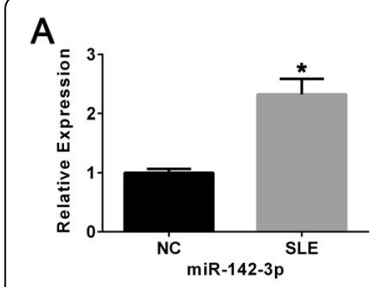

E

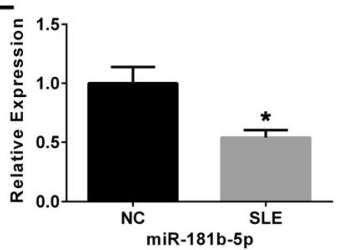

B

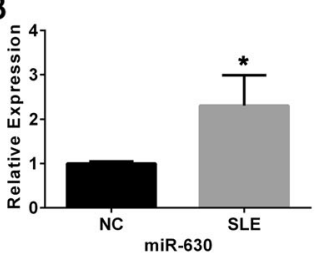

F

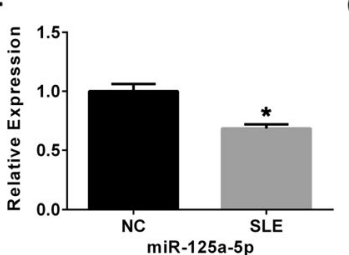

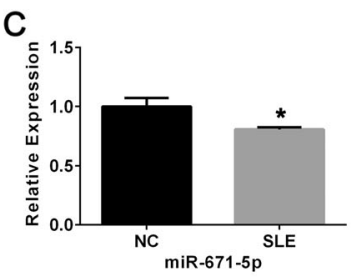

G

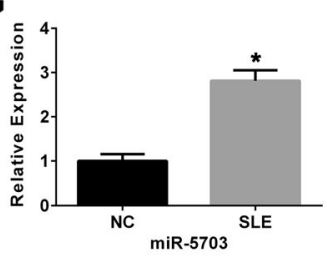

$D$

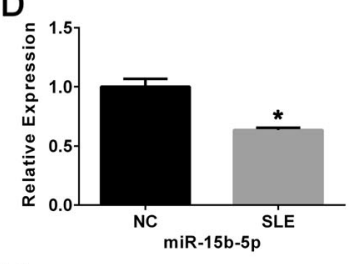

$\mathrm{H}$

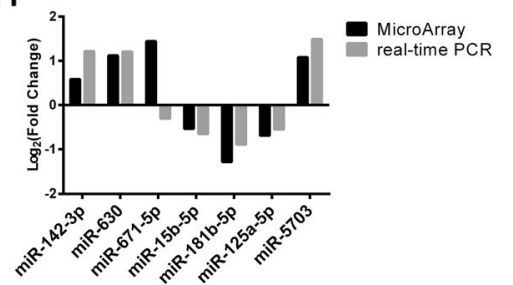

Fig. 3 Validation of selected miRNAs by qRT-PCR. The levels of miR-142-3p $\left({ }^{*} P<0.001\right)$, miR-630 ( $\left.P=0.072\right)$, miR-671-5p $\left({ }^{*} P=0.021\right)$, miR-15b-5p $\left({ }^{*} P=0.005\right)$, miR-181b-5p ( $\left.{ }^{*} P<0.001\right)$, miR-125a-5p ( $\left.{ }^{*} P<0.001\right)$, and miR-5703 $\left({ }^{*} P<0.001\right)$ were determined in LPS-activated moDCs of 15 negative controls (NCS) and 15 patients with SLE (a-g). Data were shown as mean \pm SD. The change patterns between microarray analysis and qRT-PCR were shown in (h). NC negative controls, PCR polymerase chain reaction, SLE systemic lupus erythematosus

Then, we investigated whether the expression level of miR-142-3p impacted the production of cytokines and chemokines secreted by moDCs since these soluble factors were expected to modulate $\mathrm{CD} 4^{+} \mathrm{T}$ cells-moDCs interaction. We examined the supernatants of moDCs in the VEC and LV group. We found the level of exocrine secretion of IL-6 $(1187.65 \pm 174.91 \mathrm{pg} / \mathrm{ml}, P=0.03)$ and TNF- $\alpha(262.99 \pm 25.33 \mathrm{pg} / \mathrm{ml}, P=0.013)$ were significantly increased in the supernatants of moDCoverexpressed miR-142-3p compared with the VEC group (Fig. 5a and b). As shown in Fig. $5 \mathrm{c}-\mathrm{e}$, the level of exocrine secretion of CXCL8 $(24171.17 \pm 1294.77 \mathrm{pg} / \mathrm{ml}$, $P=0.004)$, CCL2 $(412.20 \pm 27.61 \mathrm{pg} / \mathrm{ml}, P=0.014)$ and CCL5 $(126.46 \pm 4.25 \mathrm{pg} / \mathrm{ml}, P<0.001)$ was also significantly increased in LV group compared with VEC group.

\section{Elevated expression level of miR-142-3p affected} moDCs-CD4 ${ }^{+} \mathrm{T}$ cells interaction

DC-induced $\mathrm{T}$ cell priming results in a robust immune response and is vital in SLE pathogenesis [24]. To assess a possible effect of miR-142-3p on the function of moDCs reacting on $\mathrm{CD}_{4}^{+} \mathrm{T}$ cells, we first used a Transwell assay to characterize the allogeneic $\mathrm{CD}^{+} \mathrm{T}$ cells attracted by supernatant of moDCs. As presented in Fig. 6a, supernatants in the miR-142-3p overexpression group attracted significantly more $\mathrm{CD} 4 \mathrm{~T}^{+}$cell migration to the lower chamber compared with the NC and VEC group. Next, moDCs were pulsed with ovalbumin (OVA) for 2 hours and were subsequently co-cultured with allogeneic $\mathrm{CD} 4^{+}$ $\mathrm{T}$ cells. As a functional test, we compared $\mathrm{CD} 4^{+} \mathrm{T}$ cells stimulatory capacities between moDCs transfected with
A
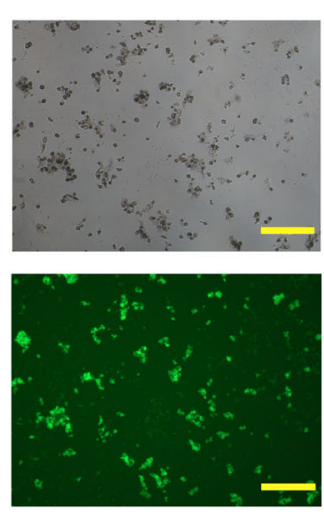

VEC
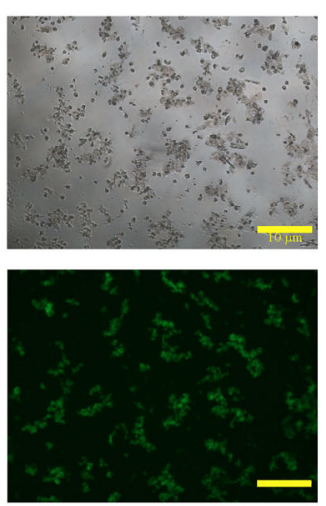

miR-142-3p LV
B

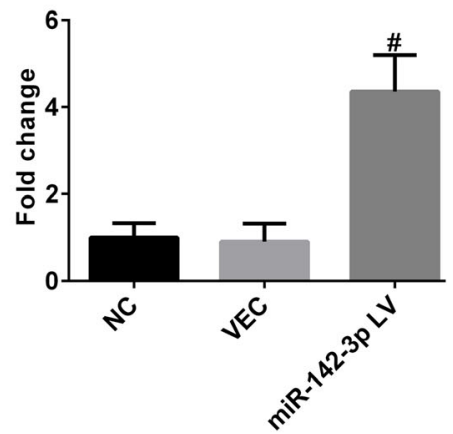

Fig. 4 Transfection efficiency of overexpressing miR-142-3p in moDCs. a Morphology and GFP fluorescence of moDCs transfected with empty lentivirus vector (VEC) and miR-142-3p overexpressing lentivirus (LV). Scale bar $=10 \mu \mathrm{m}$. b The level of miR-142-3p expressed in moDCs of negative controls (NC), VEC or LV group was assessed by quantitative real-time PCR. Data were expressed as mean \pm SD. Each experiment was conducted at least three times. ${ }^{\#} P<0.005$ versus NC and VEC 


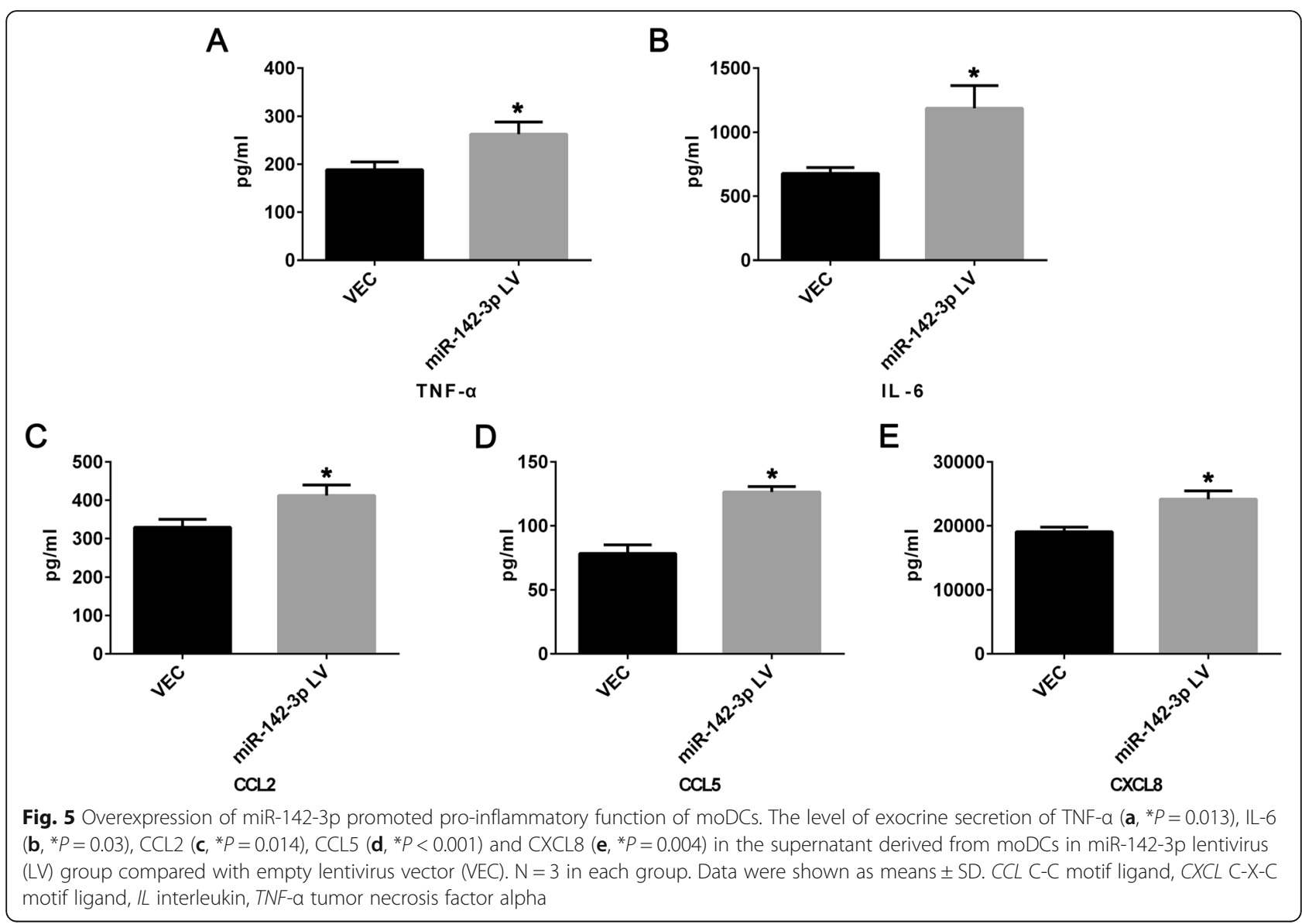

miR-142-3p overexpression lentivirus, or with empty vector, or with RPMI 1640 in an allogeneic moDCs$\mathrm{CD}^{+}{ }^{+} \mathrm{T}$ cells co-culture. The division index of $\mathrm{CD}^{+}{ }^{+} \mathrm{T}$ cells in each group was $4.60 \pm 0.19(\mathrm{NC}), 4.52 \pm 0.31$ (VEC), $4.73 \pm 0.06$ (LV) respectively. No significant differences were found among the ability of moDCs in $\mathrm{NC}, \mathrm{VEC}$ or LV group to activate $\mathrm{CD} 4^{+} \mathrm{T}$ cells proliferation, indicating that miR-142-3p might not be able to enhance $\mathrm{CD}_{4}{ }^{+} \mathrm{T}$ cells proliferation. However, the proportion of $\mathrm{CD}_{4}{ }^{+} \mathrm{CD} 25^{+}$Foxp $3^{+}$Tregs among $\mathrm{CD} 4^{+} \mathrm{T}$ cells was significantly lower when $\mathrm{CD}^{+} \mathrm{T}$ cells were co-cultured with moDCs of LV group $(1.09 \pm 0.59 \%)$ compared with NC $(4.96 \pm 0.57 \%, P=0.001)$ or VEC group $(2.86 \pm 1.03 \%, P=0.029)$ (Fig. $6 \mathrm{~b}$ and $\mathrm{c}$ ). We further examined the supernatants of co-culture medium and found the level of IL-17 was significantly higher in the LV group and the level of IL-10 was significantly decreased (Fig. 6d and e).

\section{Discussion}

SLE is an autoimmune disease characterized by autoreactive $B$ and $T$ cells [25]. There is growing evidence indicating that alterations in the function of DCs, which are APCs capable of inducing activation of naive
T cells, is related to the pathogenesis of SLE [25-27]. Given the challenges of tissue acquisition in humans, studies of DC subsets are carried out typically on DC generated in vitro from precursors. As a result, we use monocytes-derived DCs, which is a classic method to obtain enough DCs in vitro and has been successfully used as DCs according to its morphology, phenotype and function $[15,28,29]$.

In the current work, we found moDCs in patients with SLE in their active phase secreted higher level of IL-6, CCL2, and CCL5 as well as attracting more CD4 ${ }^{+} \mathrm{T}$ cells. We then performed microarray and found miR142-3p was highly expressed in human moDCs among the aberrant miRNAs in the SLE group. The function of moDCs was affected by altering the expression of miR142-3p. We further found that the pro-inflammatory cytokine IL-6, TNF- $\alpha$ and chemokine CCL2, CCL5, CXCL8 were elevated in the miR-142-3p lentivirus group. Moreover, enforced miR-142-3p in moDCs could affect moDC-CD4 ${ }^{+} \mathrm{T}$ cell interaction through inhibiting the proliferation of $\mathrm{CD}^{+} \mathrm{CD}^{+} 5^{+} \mathrm{Foxp}^{+}$Tregs and attracting more $\mathrm{CD}^{+} \mathrm{T}$ cells. Thus, we concluded miR-142-3p promoted pro-inflammatory function of moDCs and possibly contributing to the pathogenesis of SLE. 


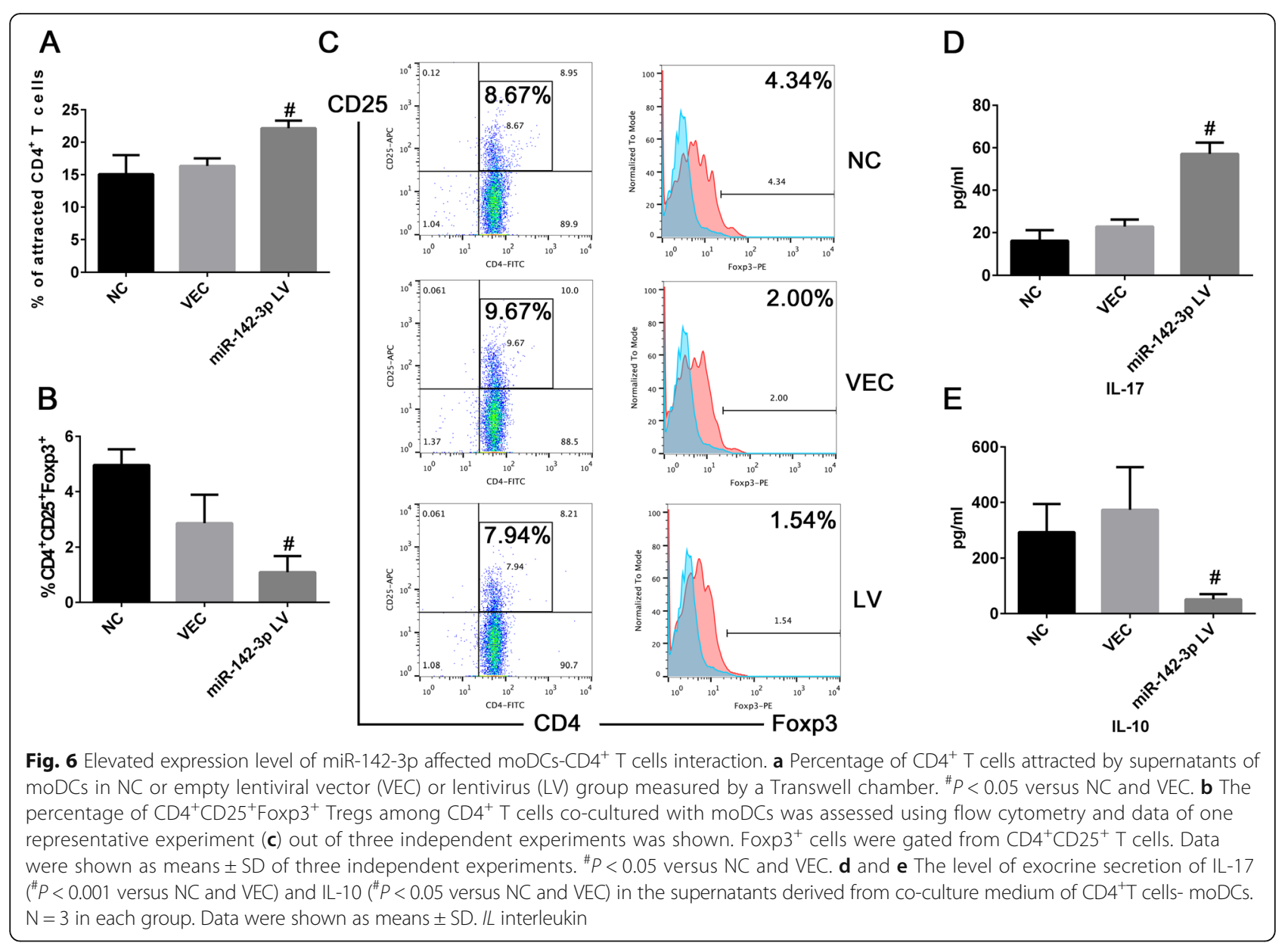

It is known from animal lupus models and from studies in patients with lupus nephritis that inflammatory chemokines, especially CCL2 and CCL5, were readily detectable in the kidney tissue and urine [30, 31]. IL-6 is significantly elevated in the body fluids of SLE patients, including serum, plasma, and cerebrospinal fluid [32-35]. Therefore, the pathogenesis of SLE involves altered cytokine production. Similarly, we have found moDCs of patients with SLE in active phase could produce higher levels of IL-6, CCL2, and CLL5, which might contribute to explain the dynamic role of these cells in disease pathogenesis. Since moDCs in our study were differentiated under the same conditions, the discrepancy between the SLE group and healthy control group was due to the intrinsic factors. It was reported monocytes in patients with SLE produced higher IL-6 and TNF- $\alpha$ [36], and presented an increase of CCL2, CXCL9, and CXCL10 mRNA expression when compared with control group [37]. Therefore, both monocytederived DCs and their parental monocytes in SLE displayed pro-inflammatory function.

miR-142 is exclusively expressed in hematopoietic cells under homeostatic conditions and has been shown to play a critical role in immune responses, such as regulating HMGB1 in THP-1 cells [38], SOCS1 in human macrophages [39], and cAMP in mice Treg cells [40]. We found miR-142-3p increased in monocyte-derived DCs from patients with SLE, which is consistent with the upregulation of miR-142-3p in B cells [41], PBMCs [42], and plasma [43] from SLE patients. In our study, functional enrichment analysis indicated that target genes of miR-142-3p (IL6ST, SPRED1, BCL2L1, STAM, IL7R, LIFR, PRLR) and (ITGB8, ITGAV, CRK, ROCK2, COL24A1, RAC1) were significantly enriched in the Jak-STAT signaling pathway and focal adhesion pathway respectively, which were important pathways for cytokines and chemokines. It has been reported these two pathways are both associated with SLE [44, 45]. Therefore, it could be speculated that miR-142-3p might play important roles in SLE by regulating their target genes that participate in these important signaling pathways.

Leukocyte migration is mediated by the interaction of a number of chemokines and their receptors [46]. These small molecules have well-defined roles in directing cell movements necessary for the initiation of $\mathrm{T}$ cell immune response, attraction of appropriate effector cells to sites 
of inflammation, and regulation of differential recruitment of T helper lymphocytes. Matured DCs could secrete an abundant source of both inflammatory and lymphoid chemokines, sustaining interaction of naive and activated $\mathrm{T}$ cells with antigen-presenting mature DCs. In our experiments, we found the level of CXCL8, CCL2, and CCL5 increased in the supernatants of moDCs with enforced expression of miR-142-3p. The three chemokines have also been reported in biological fluids from SLE [31, 47], so they might act synergistically on the chemotactic activity inducing $\mathrm{CD} 4^{+} \mathrm{T}$ cell migration. Since miR-142-3p is elevated in moDCs from SLE patients, it suggests that the upregulation of miR-142-3p in moDCs of SLE patients might be attributed to attracting more $\mathrm{CD} 4^{+} \mathrm{T}$ cells, probably involved in the pathogenesis of disease.

We also found the overexpression of miR-142-3p cause the elevation of IL- 6 and TNF- $\alpha$, leading to a decrease of $\mathrm{CD} 4^{+} \mathrm{CD} 25^{+}$Foxp $3^{+}$Tregs which are antiinflammatory, and an imbalance of IL-17 and IL-10. Treg deficiency in the periphery is sufficient to evoke chronic T cell-mediated autoimmunity and immunopathology, which has been associated with SLE [48]. The mechanisms of Treg-mediated suppression including secretion of immunosuppressive cytokines, cell contactdependent suppression, and functional modification or killing of APC [48]. It has been demonstrated in lupusprone mice that IL-6 produced by DCs inhibits Tregs [49], because Foxp $3^{+}$Treg cells lose Foxp3 expression and undergo conversion into Th17 cells under the effect of IL-6 [50]. Therefore, IL-6 triggered an immune disorder by breaking the balance between Th17 and Treg. Our results indicate that the increase of miR-142$3 \mathrm{p}$ in moDCs of SLE made the cells producing increased IL- 6 and could induce CD $4^{+} \mathrm{T}$ cells to secrete more IL-17 and less IL-10, thus leading to an imbalance of the immune response. Therefore, overexpression of miR-142-3p in moDCs suppressed the increase in Tregs, which correlated with a reduced capacity to suppress responder $\mathrm{T}$ cell proliferation and might thereby contribute to the development of SLE. Similarly, mouse dendritic cells matured by ingestion of apoptotic blebs could stimulate allogeneic $\mathrm{T}$ cells which produced IFN and especially high levels of IL-17, representing an important driving force in SLE [51].

We have suggested that elevated expression of miR$142-3 p$ is related to the pro-inflammatory function of moDCs in SLE. However, the limitation of our study is that we have not investigated the effect of decreasing the level of miR-142-3p in moDCs of SLE. Future study would focus on the exact target genes of miR$142-3 p$ in moDCs and whether downregulating miR$142-3 p$ could improve the overactive inflammation phase of SLE.

\section{Conclusion}

Taken together, our findings suggested a pro-inflammatory function of moDCs in SLE patients partially mediated by miR-142-3p: (1) microRNAs were differentially expressed and miR-142-3p was increased in moDCs of SLE patients. (2) moDCs in patients with SLE produced higher levels of some pro-inflammatory cytokines and chemokines than healthy controls. (3) Overexpressing miR-142-3p in moDCs of healthy controls promoted cytokine and chemokine production to attract more $\mathrm{CD} 4^{+} \mathrm{T}$ cells and decrease Treg expansion. These findings suggested that miR-142$3 p$ might be meaningful in the pathogenesis of SLE and could serve as a novel therapeutic target for treatment.

\section{Additional files}

Additional file 1: Table S1. The top ten enriched Gene Ontology (GO) terms for target genes of all the 18 differentially expressed miRNAs. (DOCX $72 \mathrm{~kb}$ )

Additional file 2: Table S2. The top ten enriched pathways for target genes of all the 18 differentially expressed miRNAs. (DOCX 68 kb)

\section{Abbreviations \\ 3'UTR: 3' un-translated regions; APC: Antigen-presenting cells; BSA: Bovine serum albumin; CCL: C-C motif ligand; CFSE: Carboxyfluorescein diacetate succinimidyl ester; CXCL: C-X-C motif ligand; DCs: Dendritic cells; FBS: Fetal bovine serum; FDR: False discovery rate; GM-CSF: Granulocyte-macrophage colony-stimulating factor; IL: Interleukin; LPS: Lipopolysaccharide; LV: Lentivirus; miRNAs: microRNAs; moDCs: monocyte-derived DCs; NC: Negative controls; OVA: Ovalbumin; PBMCs: Peripheral blood mononuclear cells; PBS: Phosphate- buffered saline; qRT-PCR: quantitative real-time PCR; SLE: Systemic lupus erythematosus; SOCS1: Suppressor of cytokine signaling-1; TLR: Toll-like receptor; TNF-a: Tumor necrosis factor alpha; Tregs: Regulatory T cells; VEC: Empty lentiviral vector}

\section{Acknowledgements}

Not applicable.

\section{Funding}

The research was supported by National Natural Science Foundation of China (Grant No. 81373212, 81371745, 81402605) and Development Project of Shanghai Peak Disciplines-Integrated Chinese and Western Medicine.

\section{Availability of data and materials}

The data of microarray was deposited in Gene Expression Omnibus (GEO) and the accession number was GSE79240.

\section{Authors' contributions}

WYL cultured the cells, performed the microarray and transfection experiments, carried out the flow cytometric analysis, and drafted the manuscript. LJ participated in the design of the study and drafted the manuscript. QHH participated in study design and target gene prediction, and revised the manuscript. GY performed the statistical analysis of data and revised the manuscript. DJ participated in GPCR experiments, chemotaxis assay, and manuscript drafting. LJR and ZXH collected the specimens, participated in $\mathrm{GPCR}$ experiments, and drafted the manuscript. WJ contributed to data interpretation and manuscript revision. XJH conceived of the study, participated in its design and coordination, and revised the manuscript. All authors approved the final version to be published.

Competing interests

The authors declare that they have no competing interests. 


\section{Consent for publication}

Written informed consents were obtained from the patients for publication of their individual details and any accompanying images in this manuscript. The consent form is held by the authors and is available for review by the Editor-in-Chief.

\section{Ethics approval and consent to participate}

The study was approved by the Independent Ethics Committee of Huashan Hospital and written informed consent was obtained from all subjects.

\section{Author details}

'Department of Dermatology, Huashan Hospital, Fudan University, 12 Wulumuqi Zhong Road, Shanghai 200040, People's Republic of China. Department of Neurology, Huashan Hospital, Fudan University, Shanghai, People's Republic of China. ${ }^{3}$ Department of Human Anatomy and Histoembryology, School of Basic Medical Science, Fudan University, Shanghai, People's Republic of China.

Received: 18 July 2016 Accepted: 19 October 2016 Published online: 16 November 2016

\section{References}

1. Zhu Z, Liang Z, Liany H, Yang C, Wen L, Lin Z, Sheng Y, Lin Y, Ye L, Cheng $Y$, et al. Discovery of a novel genetic susceptibility locus on $X$ chromosome for systemic lupus erythematosus. Arthritis Res Ther. 2015;17:349.

2. Feng JB, Ni JD, Yao X, Pan HF, Li XP, Xu JH, Pan FM, Xu SQ, Ye DQ. Gender and age influence on clinical and laboratory features in Chinese patients with systemic lupus erythematosus: 1,790 cases. Rheumatol Int. 2010;30(8):1017-23.

3. Patel DR, Richardson BC. Epigenetic mechanisms in lupus. Curr Opin Rheumatol. 2010;22(5):478-82.

4. Gaipl US, Munoz LE, Grossmayer G, Lauber K, Franz S, Sarter K, Voll RE, Winkler T, Kuhn A, Kalden J, et al. Clearance deficiency and systemic lupus erythematosus (SLE). J Autoimmun. 2007;28(2-3):114-21.

5. Chan VS, Nie YJ, Shen N, Yan S, Mok MY, Lau CS. Distinct roles of myeloid and plasmacytoid dendritic cells in systemic lupus erythematosus. Autoimmun Rev. 2012;11(12):890-7.

6. Teichmann LL, Ols ML, Kashgarian M, Reizis B, Kaplan DH, Shlomchik MJ. Dendritic cells in lupus are not required for activation of $T$ and B cells but promote their expansion, resulting in tissue damage. Immunity. 2010;33(6):967-78.

7. Miyara M, Amoura Z, Parizot C, Badoual C, Dorgham K, Trad S, Nochy D, Debre P, Piette JC, Gorochov G. Global natural regulatory T cell depletion in active systemic lupus erythematosus. J Immunol. 2005;175(12):8392-400.

8. Ding D, Mehta H, McCune WJ, Kaplan MJ. Aberrant phenotype and function of myeloid dendritic cells in systemic lupus erythematosus. J Immunol. 2006:177(9):5878-89.

9. Hashimi ST, Fulcher JA, Chang MH, Gov L, Wang S, Lee B. MicroRNA profiling identifies miR-34a and miR-21 and their target genes JAG1 and WNT1 in the coordinate regulation of dendritic cell differentiation. Blood. 2009;114(2):404-14.

10. Zhou $H$, Huang $X$, Cui $H$, Luo $X$, Tang $Y$, Chen $S$, Wu L, Shen N. miR155 and its star-form partner miR-155* cooperatively regulate type interferon production by human plasmacytoid dendritic cells. Blood. 2010;116(26):5885-94.

11. Lu C, Huang X, Zhang X, Roensch $K$, Cao Q, Nakayama Kl, Blazar BR, Zeng $Y$, Zhou X. miR-221 and miR-155 regulate human dendritic cell development, apoptosis, and IL-12 production through targeting of p27kip1, KPC1, and SOCS-1. Blood. 2011;117(16):4293-303.

12. Dunand-Sauthier I, Santiago-Raber ML, Capponi L, Vejnar CE, Schaad O, Irla M, Seguin-Estevez Q, Descombes P, Zdobnov EM, Acha-Orbea H, et al. Silencing of c-Fos expression by microRNA-155 is critical for dendritic cell maturation and function. Blood. 2011;117(17):4490-500

13. Kim SJ, Gregersen PK, Diamond B. Regulation of dendritic cell activation by microRNA let-7C and BLIMP1. J Clin Invest. 2013:123(2):823-33.

14. Wang $P$, Xue $Y$, Han $Y$, Lin L, Wu C, Xu S, Jiang Z, Xu J, Liu Q, Cao X. The STAT3-binding long noncoding RNA Inc-DC controls human dendritic cell differentiation. Science. 2014;344(6181):310-3.

15. Sallusto F, Lanzavecchia A. Efficient presentation of soluble antigen by cultured human dendritic cells is maintained by granulocyte/macrophage colony-stimulating factor plus interleukin 4 and downregulated by tumor necrosis factor alpha. J Exp Med. 1994;179(4):1109-18.

16. Vogelsang P, Karlsen M, Brun JG, Jonsson R, Appel S. Altered phenotype and Stat1 expression in Toll-like receptor $7 / 8$ stimulated monocyte-derived dendritic cells from patients with primary Sjogren's syndrome. Arthritis Res Ther. 2014;16(4):R166.

17. Gottschalk TA, Tsantikos E, Hibbs ML. Pathogenic inflammation and its therapeutic targeting in systemic lupus erythematosus. Front Immunol. 2015;6:550.

18. Rodriguez-Pla A, Patel P, Maecker HT, Rossello-Urgell J, Baldwin N, Bennett L, Cantrell V, Baisch J, Punaro M, Gotte A, et al. IFN priming is necessary but not sufficient to turn on a migratory dendritic cell program in lupus monocytes. J Immunol. 2014;192(12):5586-98.

19. Jiang W, Gilkeson G. Sex Differences in monocytes and TLR4 associated immune responses; implications for systemic lupus erythematosus (SLE). J Immunother Applic. 2014;1:1.

20. Nimmo R, Ciau-Uitz A, Ruiz-Herguido C, Soneji S, Bigas A, Patient R, Enver T. MiR-142-3p controls the specification of definitive hemangioblasts during ontogeny. Dev Cell. 2013;26(3):237-49.

21. Kramer NJ, Wang WL, Reyes EY, Kumar B, Chen CC, Ramakrishna C, Cantin EM, Vonderfecht SL, Taganov KD, Chau N, et al. Altered lymphopoiesis and immunodeficiency in miR-142 null mice. Blood. 2015;125(24):3720-30.

22. Mildner A, Chapnik E, Manor O, Yona S, Kim KW, Aychek T, Varol D, Beck G, Itzhaki ZB, Feldmesser E, et al. Mononuclear phagocyte miRNome analysis identifies miR-142 as critical regulator of murine dendritic cell homeostasis. Blood. 2013;121(6):1016-27.

23. Sonda N, Simonato F, Peranzoni E, Cali B, Bortoluzzi S, Bisognin A, Wang E, Marincola FM, Naldini L, Gentner B, et al. miR-142-3p prevents macrophage differentiation during cancer-induced myelopoiesis. Immunity. 2013:38(6):1236-49.

24. Fransen $\mathrm{JH}$, van der Vlag J, Ruben J, Adema GJ, Berden JH, Hilbrands LB. The role of dendritic cells in the pathogenesis of systemic lupus erythematosus. Arthritis Res Ther. 2010;12(2):207.

25. Blanco P, Palucka AK, Gill M, Pascual V, Banchereau J. Induction of dendritic cell differentiation by IFN-alpha in systemic lupus erythematosus. Science. 2001;294(5546):1540-3.

26. Scheinecker C, Zwolfer B, Koller M, Manner G, Smolen JS. Alterations of dendritic cells in systemic lupus erythematosus - phenotypic and functional deficiencies. Arthritis Rheum. 2001:44(4):856-65.

27. Sisirak V, Ganguly D, Lewis KL, Couillault C, Tanaka L, Bolland S, D'Agati V, Elkon KB, Reizis B. Genetic evidence for the role of plasmacytoid dendritic cells in systemic lupus erythematosus. J Exp Med. 2014;211(10):1969-76.

28. Chapuis F, Rosenzwajg M, Yagello M, Ekman M, Biberfeld P, Gluckman JC. Differentiation of human dendritic cells from monocytes in vitro. Eur J Immunol. 1997:27(2):431-41.

29. Pickl WF, Majdic O, Kohl P, Stockl J, Riedl E, Scheinecker C, Bello-Fernandez C, Knapp W. Molecular and functional characteristics of dendritic cells generated from highly purified CD14+ peripheral blood monocytes. J Immunol. 1996;157(9):3850-9.

30. Hasegawa H, Kohno M, Sasaki M, Inoue A, Ito MR, Terada M, Hieshima K, Maruyama H, Miyazaki J, Yoshie O, et al. Antagonist of monocyte chemoattractant protein 1 ameliorates the initiation and progression of lupus nephritis and renal vasculitis in MRL/lpr mice. Arthritis Rheum. 2003;48(9):2555-66.

31. Lit LC, Wong CK, Tam LS, Li EK, Lam CW. Raised plasma concentration and ex vivo production of inflammatory chemokines in patients with systemic lupus erythematosus. Ann Rheum Dis. 2006;65(2):209-15.

32. Abdel Galil SM, Ezzeldin N, El-Boshy ME. The role of serum IL-17 and IL-6 as biomarkers of disease activity and predictors of remission in patients with lupus nephritis. Cytokine. 2015;76(2):280-7.

33. Talaat RM, Mohamed SF, Bassyouni IH, Raouf AA. Th1/Th2/Th17/Treg cytokine imbalance in systemic lupus erythematosus (SLE) patients: correlation with disease activity. Cytokine. 2015;72(2):146-53.

34. Lyn-Cook BD, Xie C, Oates J, Treadwell E, Word B, Hammons G, Wiley K. Increased expression of Toll-like receptors (TLRs) 7 and 9 and other cytokines in systemic lupus erythematosus (SLE) patients: ethnic differences and potential new targets for therapeutic drugs. Mol Immunol. 2014;61(1):38-43.

35. Fragoso-Loyo H, Richaud-Patin Y, Orozco-Narváez A, Dávila-Maldonado L, Atisha-Fregoso Y, Llorente L, Sánchez-Guerrero J. Interleukin-6 and chemokines in the neuropsychiatric manifestations of systemic lupus erythematosus. Arthritis Rheum. 2007;56(4):1242-50.

36. Henriques $A$, Ines $L$, Carvalheiro $T$, Couto $M$, Andrade A, Pedreiro S, Laranjeira P, Morgado JM, Pais ML, da Silva JA, et al. Functional 
characterization of peripheral blood dendritic cells and monocytes in systemic lupus erythematosus. Rheumatol Int. 2012;32(4):863-9.

37. Carvalheiro T, Rodrigues A, Lopes A, Ines L, Velada I, Ribeiro A, Martinho A, Silva JA, Pais ML, Paiva A. Tolerogenic versus inflammatory activity of peripheral blood monocytes and dendritic cells subpopulations in systemic lupus erythematosus. Clin Dev Immunol. 2012;2012:934161.

38. Yuan Z, Luo G, Li X, Chen J, Wu J, Peng Y. PPARY inhibits HMGB1 expression through upregulation of miR-142-3p in vitro and in vivo. Cell Signal. 2016;28(3):158-64

39. Su S, Zhao Q, He C, Huang D, Liu J, Chen F, Chen J, Liao J-Y, Cui X, Zeng Y, et al. miR-142-5p and miR-130a-3p are regulated by IL-4 and IL-13 and control profibrogenic macrophage program. Nat Commun. 2015;6:8523.

40. Huang B, Zhao J, Lei Z, Shen S, Li D, Shen G-X, Zhang G-M, Feng Z-H. miR-142$3 p$ restricts cAMP production in CD4(+)CD25(-) T cells and CD4(+)CD25(+) T(REG) cells by targeting AC9 mRNA. EMBO Rep. 2009:10(2):180-5.

41. Te JL, Dozmorov IM, Guthridge JM, Nguyen KL, Cavett JW, Kelly JA, Bruner GR, Harley JB, Ojwang JO. Identification of unique microRNA signature associated with lupus nephritis. PLoS One. 2010;5(5):e10344.

42. Dai Y, Huang YS, Tang M, Lv TY, Hu CX, Tan YH, Xu ZM, Yin YB. Microarray analysis of microRNA expression in peripheral blood cells of systemic lupus erythematosus patients. Lupus. 2007;16(12):939-46.

43. Carlsen AL, Schetter AJ, Nielsen CT, Lood C, Knudsen S, Voss A, Harris CC, Hellmark T, Segelmark M, Jacobsen S, et al. Circulating microRNA expression profiles associated with systemic lupus erythematosus. Arthritis Rheum. 2013;65(5):1324-34.

44. Tang Y, Ma X, Zhang H, Gu Z, Hou Y, Gilkeson GS, Lu L, Zeng X, Sun L. Gene expression profile reveals abnormalities of multiple signaling pathways in mesenchymal stem cell derived from patients with systemic lupus erythematosus. Clin Dev Immunol. 2012;2012:826182.

45. Kawasaki M, Fujishiro M, Yamaguchi A, Nozawa K, Kaneko H, Takasaki Y, Takamori K, Ogawa H, Sekigawa I. Possible role of the JAK/STAT pathways in the regulation of $\mathrm{T}$ cell-interferon related genes in systemic lupus erythematosus. Lupus. 2011;20(12):1231-9.

46. Sallusto F, Lanzavecchia A. Understanding dendritic cell and T-lymphocyte traffic through the analysis of chemokine receptor expression. Immunol Rev. 2000;177:134-40.

47. Laborde EA, Vanzulli S, Beigier-Bompadre M, Isturiz MA, Ruggiero RA Fourcade MG, Catalan Pellet AC, Sozzani S, Vulcano M. Immune complexes inhibit differentiation, maturation, and function of human monocytederived dendritic cells. J Immunol. 2007;179(1):673-81.

48. Sakaguchi S, Yamaguchi T, Nomura T, Ono M. Regulatory T cells and immune tolerance. Cell. 2008;133(5):775-87.

49. Wan S, Xia C, Morel L. IL-6 produced by dendritic cells from lupus-prone mice inhibits CD4 + CD25+ T cell regulatory functions. J Immunol. 2006;178(1):271-9.

50. Komatsu N, Okamoto K, Sawa S, Nakashima T, Oh-hora M, Kodama T, Tanaka S, Bluestone JA, Takayanagi H. Pathogenic conversion of Foxp3+ T cells into TH17 cells in autoimmune arthritis. Nat Med. 2014;20(1):62-8.

51. Fransen JH, Hilbrands LB, Ruben J, Stoffels M, Adema GJ, van der Vlag J, Berden JH. Mouse dendritic cells matured by ingestion of apoptotic blebs induce T cells to produce interleukin-17. Arthritis Rheum. 2009;60(8):2304-13.

\section{Submit your next manuscript to BioMed Central and we will help you at every step:}

- We accept pre-submission inquiries

- Our selector tool helps you to find the most relevant journal

- We provide round the clock customer support

- Convenient online submission

- Thorough peer review

- Inclusion in PubMed and all major indexing services

- Maximum visibility for your research

Submit your manuscript at www.biomedcentral.com/submit

Biomed Central 